\title{
Análisis de las habilidades del manejo del grupo en un programa de educación afectivo-sexual para DFI ${ }^{1}$
}

\author{
María Dolores Gil-Llario \\ Departamento de Psicología Evolutiva y de la Educación, Universitat de València, \\ Valencia, España \\ dolores.Gil@uv.es \\ Verónica Estruch-García \\ Departamento de Psicología Evolutiva y de la Educación, Universitat de València, \\ Valencia, España \\ vesgar2@alumni.uv.es \\ Olga Fernández-García \\ Departamento de Psicología Evolutiva y de la Educación, Universitat de València, \\ Valencia, España \\ Olga.Fernandez-Garcia@uv.es
}

\begin{abstract}
RESUMEN
Las habilidades de manejo de grupo que presenten los profesionales que imparten los programas de educación afectivo-sexual para personas con discapacidad intelectual son esenciales a fin de dirigir las sesiones hacia los objetivos establecidos. Por ello, el objetivo del presente trabajo es explorar las incidencias producidas durante la aplicación del Programa SALUDIVERSEX, en función del tipo de contenido de la sesión, analizando las habilidades de manejo del grupo de los 29 profesionales (90\% mujeres y $10 \%$ hombres) que aplicaron este programa. Los resultados sugieren que las conductas inapropiadas de los/as usuarios/as son las incidencias más frecuentes tanto en las sesiones que trabajan habilidades $(58,3 \%)$ como las que trabajan actitudes $(53,8 \%)$. Sin embargo, la baja participación se produce con mayor frecuencia en las sesiones que trabajan actitudes $(30,7 \%)$, lo que podría deberse a la percepción de utilidad de las temáticas trabajadas en estas sesiones. Por otra parte, el surgimiento de emociones como vergüenza y/o nervosismo es más común en sesiones dedicadas a trabajar habilidades (25\%). Respecto a las habilidades de los profesionales, en la mayoría de las ocasiones, responden adecuadamente a los problemas surgidos. No obstante, el 28,5\% de las veces que tiene lugar una conducta inadecuada, el profesional no reacciona correctamente; el 50\% de las veces que algún usuario/a o el grupo presenta baja participación, el profesional ignora a la persona o acorta el contenido de la sesión; y el $42 \%$ de las veces que aparecen

\footnotetext{
${ }^{1}$ Esta investigación ha sido realizada gracias a un proyecto de investigación financiado por el Programa Estatal de I+D+i de la Convocatoria 2018 Orientada a los Retos de la Sociedad del Ministerio de Ciencia. Innovación y Universidades (Proyecto RTI2018-095538-B-I00).
} 
emociones que pueden interferir en el desarrollo de las actividades, los profesionales no gestionan de forma oportuna la situación. Estos resultados resaltan la necesidad de abordar las habilidades de los profesionales para manejar el grupo, a fin de que este aspecto no afecte a la efectividad de las intervenciones.

Palabras clave: SALUDIVERSEX; discapacidad intelectual; manejo del grupo; profesionales; habilidades; actitudes.

\begin{abstract}
The professionals who implement affective-sexual education programs for intellectual disabilities people need to have group management skills to directing the sessions towards the established objectives. Therefore, the purpose of this study was to explore the incidents that occurred in the application of the SALUDIVERSEX Program, depending on the type of content of the session (skills or attitudes) and analyzing the management skills of the group in 29 professionals (90\% were women and $10 \%$ were men). The results suggest inappropriate behaviors of users are the most frequent incidents both in the sessions to work skills $(58,3 \%)$ and attitudes $(53.8 \%)$. However, the low participation is more often in the sessions that work attitudes $(30,7 \%)$. This could be due to user perceptions of usefulness of the topics worked in these sessions. On the other hand, the emergence of emotions is more usual in skills sessions (25\%). Regarding the skills of professionals, in the most cases, they respond adequately to the problems that arise. However, they don't react correctly to inappropriate behavior in the $28,5 \% \%$ of the time, and they don't know to manage emotions in the $42 \%$ of the time, and they don't react correctly to low participation in the $50 \%$ of the time. These results highlight the need to address the group management skills of professionals so that this aspect does not affect the effectiveness of interventions.
\end{abstract}

Key words: SALUDIVERSEX; intellectual disabilities; manage of group; professionals; skills; attitudes. 


\section{INTRODUCCIÓN}

La sexualidad es un aspecto inherente del ser humano que está presente a lo largo de todo el ciclo vital (Morell-Mengual et al., 2017), por lo que resulta difícil abordar el desarrollo integral del individuo sin tener en cuenta esta dimensión. En esta línea, las personas con diversidad funcional intelectual (DFI) han sufrido tradicionalmente una gran discriminación como consecuencia de las creencias erróneas extendidas en la sociedad acerca de su desarrollo sexual (Verdugo et al., 2002) que les categorizan como personas asexuales o con dificultades para regular sus impulsos sexuales. No obstante, en 2006 la Convención Internacional sobre los Derechos de las Personas con Discapacidad motivó un mayor reconocimiento del derecho de este colectivo a vivir la sexualidad de forma libre y adaptada a sus capacidades. Esto ayudó a que, no solo la sociedad, sino también los familiares de las personas con DFI, mostraran mayor tolerancia hacia la expresión de la sexualidad de este colectivo, manifestando cada vez mayor consciencia acerca de la necesidad que tienen éstos de recibir información sobre salud sexual. No obstante, los padres de las personas con DFI continúan mostrando incerteza, por el temor a que sus hijos/as no sean capaces de controlar sus impulsos sexuales (López, 2011), aunque este intento de protegerlos únicamente dificulte su desarrollo sexual y aumente el riego de que se expongan a enfermedades de transmisión sexual y embarazos no deseados (Gil-Llario et al., 2019) Además, esto puede contribuir a la baja capacidad que tienen para identificar situaciones de riesgo, lo que les convierte en potenciales víctimas de abusos sexuales y explica la mayor prevalencia de este tipo de sucesos en este grupo frente a la población general (Tomsa et al., 2021)

Los conocimientos sobre sexualidad se adquieren mediante la información reportada en las iniciativas de educación sexual, transmitida por las personas del entorno y mediante las experiencias vividas con los iguales. Actualmente, un mayor porcentaje de personas con DFI ha recibido educación afectivo-sexual, sin embargo, muchas de las propuestas formativas no ofrecen suficiente información o no tienen en cuenta las características de este colectivo (Navarro et al., 2010). En esta línea, Jahoda \& Pownall (2014) hallaron que los conocimientos y las fuentes de información sobre sexualidad que tenían los participantes sin DFI eran mayores que las de la población con DFI. Otro aspecto a tener en cuenta son las limitaciones que presentan las personas con DFI para desarrollar habilidades sociosexuales a partir de las experiencias sociales vividas (Campos, 2003). En consecuencia, esta falta de conocimientos y habilidades restringe la capacidad de disfrutar de forma saludable y positiva de la sexualidad. En esta línea, la investigación de Gil-Llario et al. (2018) concluye que, si bien las prácticas sexuales de las que disfruta este colectivo no difieren de las de la población general, predomina en ellos el sentimiento de insatisfacción sexual.

Detectadas estas limitaciones en el desarrollo sexual que aún presentan las personas con DFI y dada la falta de programas de educación afectivo-sexual integrales adaptados a las necesidades de este colectivo, Gil-Llario et al. (2019) desarrollan el Programa SALUDIVERSEX de educación afectivo sexual para adultos con diversidad intelectual, que ha sido diseñado para ser aplicado por los profesionales que trabajan con este colectivo, dada la relación de confianza que tienen con los usuarios de los centros. Este programa está estructurado en 16 sesiones a través de las cuales se ofrece información básica que permite mejorar el nivel de conocimiento, potenciar el desarrollo de 
habilidades y estrategias de interacción, y fomentar las actitudes positivas hacia la sexualidad. A pesar de que en todas ellas se abordan necesariamente los tres componentes (conocimientos, habilidades y actitudes) en función del contenido de las mismas se establece el foco en uno de ellos u en otro. Así mismo, dada la importancia de aportar evidencia empírica sobre la efectividad de estas intervenciones (Aceldo et al., 2006), los autores establecieron la metodología de evaluación teniendo en cuenta los hallazgos recogidos en la literatura sobre los factores moduladores de la efectividad de los programas de educación afectivo sexual para personas con diversidad funcional. En esta línea, Gonzálvez et al. (2018) hallaron que la efectividad del programa es mayor cuando es aplicado por profesionales con alto y medio nivel de formación. Así mismo, Buston et al. (2002) identificaron la capacidad y motivación de los profesionales como un factor que obstaculizó la implementación rigurosa de su programa.

Por todo ello, con la finalidad de detectar los problemas más comunes que surgen durante la realización de estas formaciones con dicho colectivo y esclarecer la formación y habilidades específicas que deberían presentar los profesionales que llevan a cabo las intervenciones de educación afectivo sexual, el presente trabajo se plantea el objetivo de explorar las incidencias ocurridas durante el desarrollo del Programa SALUDIVERSEX, en función del contenido de la sesión, así como identificar las habilidades del manejo del grupo que tienen los profesionales que aplicaron el mismo.

\section{MÉTODO}

\section{Participantes}

La muestra está compuesta por 29 profesionales que implementaron el programa SALUDIVERSEX en el centro ocupacional de la Comunidad Valenciana en el que trabajaban. Respecto al género de los mismos, el $90 \%$ eran mujeres, mientras que el $10 \%$ eran hombres. En la mayoría de las ocasiones (95\%) ejercían como psicólogos en el recurso, mientras un pequeño porcentaje eran maestros de taller (5\%).

\section{Procedimiento.}

A partir de la demanda realizada al grupo de investigación SALUSEX de la cooperativa COPAVA dedicada a la atención a personas con DFI, se llevó a cabo la formación sobre la implementación del programa SALUDIVERSEX a los profesionales de 17 de los centros ocupacionales que forman parte de esta red de servicios. Una ver recogidos los permisos por parte de los/as directores/as de los diferentes recursos y de los padres de los/as usuarios/as, dos profesionales de cada centro ocupacional se beneficiaron de dicha formación que fue impartida por personas expertas en educación afectivo-sexual del equipo SALUSEX, quienes habían participado en la elaboración del programa. Este curso se realizó de forma sincrónica a la aplicación del programa por parte de los profesionales del centro ocupacional, por lo que durante el mismo se instruyó a estos en el procedimiento a seguir para aplicar el programa SALUDIVERSEX: el desarrollo de cada una de las sesiones, la evaluación en las diferentes fases (antes, durante y después de la intervención) y los instrumentos de evaluación a emplear. Así mismo, los profesionales recogieron las incidencias ocurridas en cada sesión que habían dificultado el desarrollo normal de la misma, empleando una hoja de registro que cumplimentaban al 
finalizar cada una de las sesiones y en la que describían el tipo de suceso ocurrido, la acción emprendida para solventarlo y las consecuencias de dicha acción.

\section{Análisis de datos}

En base a los datos recogidos en los informes de incidencias, se ha extraído la frecuencia con la que los profesionales informaban de cada una de las incidencias registradas que habían dificultado el desarrollo normal de las sesiones, teniendo en cuenta si se trataba de sesiones en las que se abordaba el desarrollo de habilidades o se trabajaban sus actitudes. Tras este análisis, encontramos que las incidencias más frecuentes son: las conductas disruptivas de los/as usuarios/as durante el desarrollo de la sesión (comportamientos disruptivos o comentarios impertinentes), las situaciones en las que algún/a usuario/a o el grupo completo ha presentado baja participación por falta de atención o motivación; $y$, por último, aquellas en las que un/a usuario/a ha experimentado emociones como vergüenza, enfado o nerviosismo (emocionalidad). Una vez detectadas las incidencias más comunes, se analizaron las habilidades de los profesionales en el manejo del grupo, atendiendo a la adecuación de las estrategias empleadas para solventar cada tipo de acontecimiento y registrando el porcentaje de veces que se llevó a cabo cada una de estas.

\section{RESULTADOS}

Cuando analizamos las incidencias que presentan mayor prevalencia, teniendo en cuenta el tipo de contenido de las sesiones, encontramos que, como se puede observar en la tabla 1, tanto en las sesiones centradas en el desarrollo de habilidades como las focalizadas en trabajar las actitudes, las conductas inapropiadas son los incidentes más reportados por los profesionales $(58.3 \%$ en las sesiones sobre habilidades y $53.8 \%$ en las sesiones sobre actitudes). Seguidamente, en las sesiones dedicadas al desarrollo de habilidades, la emocionalidad, que hace referencia al surgimiento de emociones de vergüenza, nerviosismo o enfado desproporcionados por parte de los/as usuarios/as, presenta el segundo porcentaje más alto $(25 \%)$, y, por último, la baja participación fue la incidencia menos frecuente $(16,7 \%)$. Mientras que en las sesiones que se trabajan actitudes, los sucesos reportados en segundo lugar son los relacionados con la baja participación $(30,7 \%)$ y, la emocionalidad fue la incidencia menos frecuente $(15,4 \%)$.

Por otra parte, como se recoge en la tabla 1, en relación con las reacciones de los/as profesionales frente a los problemas que suceden durante las sesiones, estos responden adecuadamente el $60 \%$ de las veces que acontece una incidencia, favoreciendo la implicación de los/las usuarios/as durante las diferentes sesiones (por ejemplo: reconduciendo las conductas inadecuadas de un/a usuario/a, adecuando el clima del aula y validando las emociones que presentan algunos/as frente a las actividades o temáticas). No obstante, se observa que realizan, en mayor medida, estrategias inadecuadas ante la baja participación (50\%) y la emocionalidad (42\%) respecto a las situaciones en la que se producen conductas inadecuadas $(28.2 \%)$. Concretamente, ante la desmotivación de un/a usuario/a o del grupo, han ignorado al usuario el 33,3\% de las veces que ha ocurrido o han reducido el contenido de la sesión en el 16,7\% de las ocasiones. Por otra parte, cuando algún/a usuario/a ha mostrado una reacción emocional que dificultaba el desarrollo 
normal de la sesión, las medidas emprendidas se han limitado en el 14\% de las ocasiones a preguntar el motivo, a ignorar al usuario el 14\% de las veces también o evitar las actividades que se asociaban a tales sucesos (14\%). Y, por último, en los momentos en los que un/a usuario/a ha realizado algún comportamiento inadecuado, en el 14,1\% de las ocasiones los profesionales se han limitado a preguntar, o han ignorado al usuario/a. 


\section{Tabla 1.}

Incidencias y reacción de los profesionales.

Contenido sesiones Cuestión

Conducta inadecuada (comportamiento disruptivo, comentarios impertinentes) $(58,3 \%)$

Habilidades Baja participación (somnolencia, distracciones, etc.) $(16,7 \%)$

Emocionalidad (vergüenza, emociones negativas, nerviosismo, etc.) $(25 \%)$

Conducta inadecuada (comportamiento disruptivo, comentarios impertinentes) $(53,8 \%)$

Baja participación

Actitudes (somnolencia, distracciones) $(30,7 \%)$

Emocionalidad (vergüenza, emociones negativas, nerviosismo, etc.) $(15,4 \%)$

\section{¿Cómo reacciona el profesional?}

Correcto/ Incorrecto

Nada, continuar con la sesión.

Reconducir, calmar al usuario e incitar a reflexionar.

Incorrecto

Correcto

Cambio de parejas para evitar confrontaciones.

Correcto

Cambiar el tono y ritmo de voz, encender las luces y hacer preguntas.

No completar la sesión y retomarla en otro momento.

Correcto

Correcto

Dejarle que no participe y se mantenga pasivo durante la sesión. Incorrecto

Reducir el número de intervenciones (rol-playing).

Incorrecto

Validar sus emociones resaltando que es normal sentirse incómodo al principio. Correcto Normalizar su reacción y motivarles para que participen.

Dar un aviso al/a la usuario/a y proponer una técnica para regularse.

Reconducir, incitar a reflexionar y motivarles.

Recordar que una de las normas del taller es respetar a los demás.

Correcto

Correcto

Correcto

Correcto

Preguntarle cómo se ha enterado de ese aspecto íntimo del compañero.

Incorrecto

Reconducir la sesión incluyendo más ejemplos prácticos.

Condensar la información y acortar la sesión.

Dejarle que no participe y se mantenga pasivo durante la sesión.

Ofrecerle la oportunidad de abandonar el aula.

Preguntar al/a la usuario/a el motivo de esas emociones.

Tratar con naturalidad ignorando el tema.

Realizar un ejercicio para fomentar la empatía, incluyendo ejemplos más cercanos y reconduciendo hacia emociones positivas.
Correcto

Incorrecto

Incorrecto

Correcto

Incorrecto

Incorrecto

Correcto 


\section{DISCUSIÓN}

Los resultados del presente estudio sugieren que las conductas inapropiadas de los/as usuarios/as durante el desarrollo de las sesiones son las incidencias más frecuentes tanto en las sesiones que trabajan actitudes como las que trabajan habilidades. Sin embargo, la baja participación se produce el doble de veces en las sesiones que trabajan actitudes que en las enfocadas en trabajar habilidades, lo que podría deberse a la percepción de utilidad de las temáticas trabajadas en estas sesiones. Por otra parte, el surgimiento de emociones como vergüenza y/o nervosismo es más común en sesiones dedicadas a trabajar habilidades, lo que podría estar relacionado con la exposición pública durante las dinámicas de role playing muy habituales en este tipo de sesiones.

Respecto a las habilidades de los/as profesionales, nuestros resultados sugieren que, en la mayoría de las ocasiones, responden adecuadamente a los problemas surgidos. No obstante, en relación a las reacciones inadecuadas parece que presentan más dificultades a la hora de implicar a aquellos/as usuarios/as que no están motivados en las actividades de la sesión, y para gestionar las emociones desreguladas de los/as participantes durante el desarrollo de la misma, ya que optan por ignorar a los/as usuarios/as o reducir el contenido de la sesión dejando aspectos sin tratar. Estos hallazgos están en la línea de lo expuesto por Buston et al. (2002), quienes identificaron que aquellos profesionales con bajas capacidades alteraron la estructura del programa, disminuyendo las actividades o partiendo las sesiones en dos momentos. No obstante, cuando el acontecimiento que interfiere en la sesión son los comportamientos disruptivos de los participantes, los profesionales emplean estrategias adecuadas para reconducir esta conducta, siendo muy pocas las ocasiones en las que no emprenden ninguna medida. Estos resultados podrían deberse a que los profesionales tienen una mayor destreza para reconducir estos comportamientos, ya que están más acostumbrados abordar este tipo de problemas en el resto de las actividades en las que tratan con los/as usuarios/as.

\section{CONCLUSIONES}

Estos resultados resaltan la necesidad de abordar los conocimientos, habilidades y las actitudes de los profesionales que imparten los programas de educación afectivo-sexual para personas con DFI a través de los cursos de formación de formadores. En dichas formaciones se les debe proveer a los profesionales de estrategias de manejo del grupo, así como habilidades para abordar las situaciones conflictivas más comunes en este tipo de intervenciones. Así mismo, será interesante que durante las formaciones, ellos conozcan qué tipo de incidencias son más habituales en cada tipo de sesión dependiendo del tipo de contenido que se trabaja principalmente (conocimientos, habilidades y actitudes). Con todo lo expuesto queremos incidir en la importancia de que las personas encargadas de implementar los programas de educación afectivo-sexual en este colectivo sean rigurosamente preparadas para dicho propósito, a fin de que no se vea afectada la efectividad de las intervenciones por la subjetividad del formador. 


\section{REFERENCIAS}

Aceldo, M. A., Aguado, A. L., \& Arias, B. (2006). Eficacia de un programa de educación sexual en jóvenes con discapacidad intelectual. Análisis y Modificación de Conducta, 32(142), 217-239.

Buston, K., Wight, D., Hart, G., \& Scott, S. (2002). Implementation of a teacher-delivered sex education programme: obstacles and facilitating factors. Health Education Research, 17(1), 59-72. https://doi.org/10.1093/her/17.1.59

Campos, I. (2003). Aspectos de las relaciones afectivas y sexuales en personas con discapacidad intelectual. Informacio Psicologica, 83, 15-19.

Gil-Llario, M. D., Ballester-Arnal, R., Caballero-Gascón, L., \& Escalera, C. (2019). Programa SALUDIVERSEX. Programa de educación afectivo-sexual para adultos con diversidad funcional intelectual. Pirámide.

Gil-Llario, M. D., Morell-Mengual, V., Ballester-Arnal, R., \& Díaz-Rodríguez, I. (2018). The experience of sexuality in adults with intellectual disability. Journal of Intellectual Disability Research, 62(1), 72-80. https://doi.org/10.1111/jir.12455

Gonzálvez, C., Fernández-Sogorb, A., Sanmartín, R., Vicent, M., Granados, L., \& GarcíaFernández, J. M. (2018). Efficacy of Sex Education Programs for People with Intellectual Disabilities: A Meta-Analysis. Sexuality and Disability, 36(4), 331-347. https://doi.org/10.1007/s11195-018-9545-9

Jahoda, A., \& Pownall, J. (2014). Sexual understanding, sources of information and social networks; the reports of young people with intellectual disabilities and their nondisabled peers. Journal of Intellectual Disability Research, 58(5), 430-441. https://doi.org/10.1111/jir.1204

López, F. (2011). Guía para el desarrollo de la afectividad y de la sexualidad de las personas con discapacidad intelectual. Consejería de Familia e Igualdad de Oportunidades Gerencia de Servicios Sociales.

Morell-Mengual, V., Gil-Llario, M. D., Díaz-Rodríguez, I., \& Caballero-Gascón, L. (2017). Actitudes de padres, profesionales y población general hacia la sexualidad de las personas con discapacidad física e intelectual. International Journal of Developmental and Educational Psychology, 4(1), 173-184. https://doi.org/10.17060/ijodaep.2017.n1.v4.1040

Navarro, Y., Torrico, E., \& López, M. J. (2010). Programa de intervención psicosexual en personas con discapacidad intelectual. Educación y Diversidad, 4(2), 75-92.

Tomsa, R., Gutu, S., Cojocaru, D., Gutiérrez-bermejo, B., Flores, N., \& Jenaro, C. (2021). Prevalence of sexual abuse in adults with intellectual disability: Systematic review and meta-analysis. International Journal of Environmental Research and Public Health, 18(4), 1-17. https://doi.org/10.3390/ijerph18041980

Verdugo, M. Á., Alcedo, M., Bermejo, B., \& Aguado, A. (2002). El abuso sexual en personas con discapacidad intelectual. Psicothema, 14(1), 124-129. 